\title{
Fertility and COVID-19 vaccination
}

\author{
Iosif M. Gershteyn ${ }^{1,2}{ }^{*}$ \\ ${ }^{1}$ Ajax Biomedical Foundation, Newton, MA 02461, USA \\ ${ }^{2}$ ImmuVia LLC, Waltham, MA, USA
}

*Correspondence: Iosif M. Gershteyn, Ajax Biomedical Foundation, Newton, MA 02461, USA. iosifmg@bu.edu

Academic Editor: Satish Kumar Gupta, Indian Council of Medical Research, India

Received: August 30, 2021 Accepted: December 1, 2021 Published: February 11, 2022

Cite this article: Gershteyn IM. Fertility and COVID-19 vaccination. Explor Immunol. 2022;2:1-8. https://doi.org/10.37349/ ei.2022.00032

\begin{abstract}
One of the most troubling developments of 2021 has been the number of fertile-age women who have been led to believe that mRNA vaccines against severe acute respiratory syndrome coronavirus-2 [SARS-CoV-2, coronavirus disease 2019 (COVID-19)] can cause infertility via cross-reactivity of immune response. Specifically, cross-reactivity of developed antibodies to syncytin-1, a protein found in human cell fusion, placentation and recently identified in the envelope gene of a human endogenous defective retrovirus, HERV-W (see "Syncytin is a captive retroviral envelope protein involved in human placental morphogenesis". Nature. 2000;403:785-9. doi: 10.1038/35001608). The mechanism, evidence, and evaluation of the claim is presented concluding in a rejection due to lack of evidence.
\end{abstract}

\section{Keywords}

Fertility, messenger RNA, vaccine, coronavirus disease 2019, syncytin-1

\section{The claim}

The claim is attributable to Dr. Michael Yeadon and Dr. Wolfgang Wodarg's Stay of Action petition to the European Medical Agency (EMA) on the Phase III clinical trial of coronavirus disease 2019 (COVID-19) vaccine BNT162b by Pfizer/BioNTech [1] (now authorized by the EMA [2] and fully approved by the Food and Drug Administration [3]). The claim that has reached popular consciousness and spread via the internet is in section XI of the petition.

"Several vaccine candidates are expected to induce the formation of humoral antibodies against spike proteins of severe acute respiratory syndrome coronavirus-2 (SARS-CoV-2). Syncytin-1 (see 'Response to nCoV2019 against backdrop of endogenous retroviruses', http://virological.org/t/response-toncov2019-against-backdrop-of-endogenous-retroviruses/396), which is derived from human endogenous retroviruses (HERV) and is responsible for the development of a placenta in mammals and humans and is therefore an essential prerequisite for a successful pregnancy, is also found in homologous form in the spike proteins of SARS viruses. There is no indication whether antibodies against spike proteins of SARS viruses would also act like anti-syncytin-1 antibodies. However, if this were to be the case this would then also 
prevent the formation of a placenta which would result in vaccinated women essentially becoming infertile. To my knowledge, Pfizer/BioNTech has yet to release any samples of written materials provided to patients, so it is unclear what, if any, information regarding (potential) fertility-specific risks caused by antibodies is included."

The mechanism of this hypothetical action can be described in this sequence:

1) The COVID-19 vaccine generates an immune response to the spike glycoprotein (Figure 1).

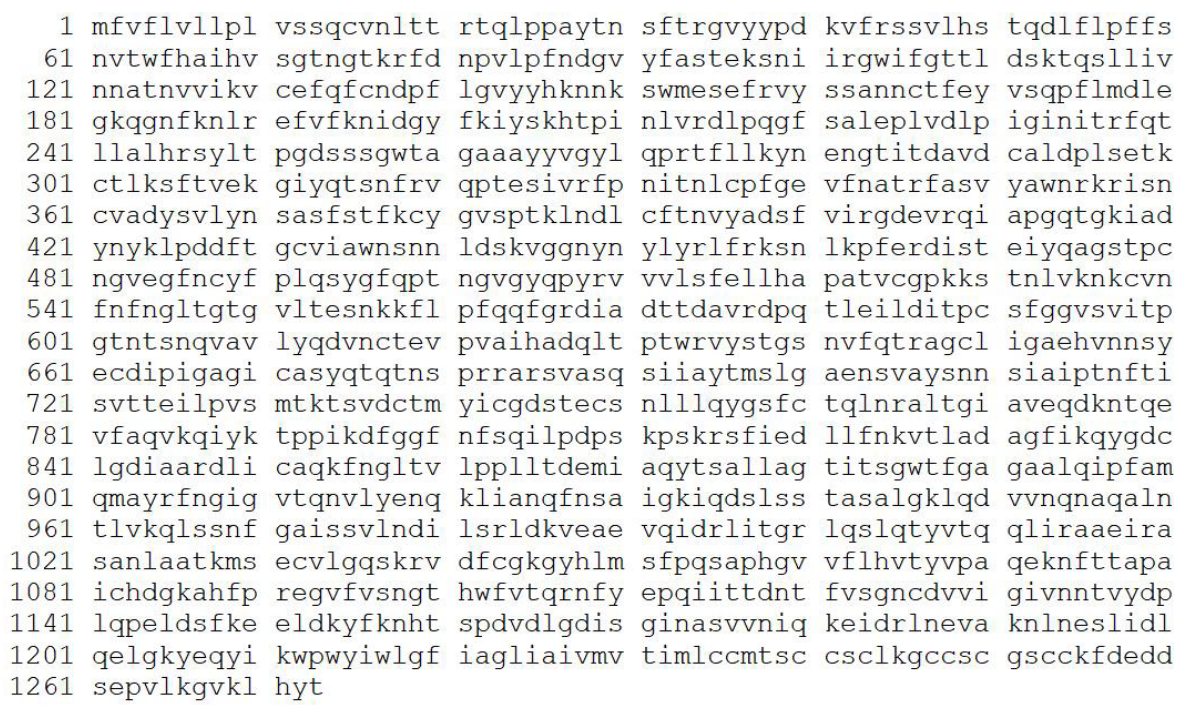

Figure 1. Amino acid sequence of spike glycoprotein in SARS-CoV-2 (COVID-19)

2) Due to similarity (Figure 2) between the spike glycoprotein and syncytin-1 (Figure 3); generated antibodies or other immune changes (likely T-cell-mediated) will confuse the two proteins (cross-react) and therefore attack syncytin-1 expressing cells.

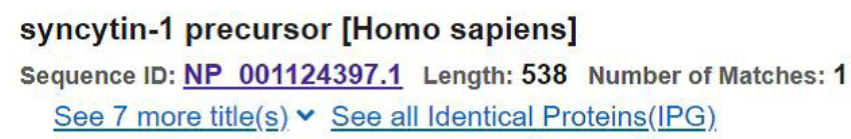

\begin{tabular}{lllll}
\hline Score & Expect & Identities & Positives & Gaps \\
24.1 bits(61) & 0.024 & $19 / 59(32 \%)$ & $31 / 59(52 \%)$ & $3 / 59(5 \%)$ \\
\hline
\end{tabular}

Query 908 GIG-VTQNVLYENQKLIANQFNSAIGKIQDSLSSTASALGKLQDVVNQNAQALNTLVKQ 965

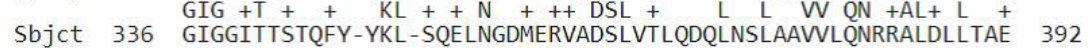

Figure 2. Overlap regions of syncytin-1 and spike glycoprotein

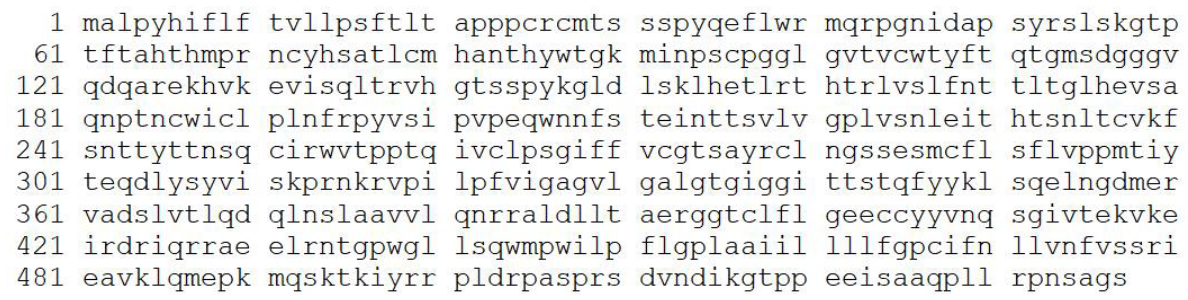

Figure 3. Amino acid sequence of syncytin-1 (human)

3) Syncitin-1 is involved with placentation and if damaged will prevent ability of the placenta to form or function.

4) If the placenta cannot function, then the affected woman is functionally infertile as she cannot support a healthy pregnancy. 


\section{Molecular mechanism}

This link between immune response to the spike glycoprotein (a 1273 [4] amino acid long protein targeted by COVID vaccines) and placental protein (syncytin-1, a 538 [5] amino acid long protein) is not substantiated by the evidence.

To understand the flaw in evidence, we must define some (limited) aspects of immune system functioning:

1) Proteins are molecules composed of amino acids, analogous to each amino acid acting as a character (letter) in a sentence (excluding spaces, as there is no space between amino acid chains).

2) The median length of a human protein is about 248 amino acids long-a sentence almost exactly the length of the first sentence of this article ( 262 characters, not counting spaces). The longest human protein is Titin at 27,000 [6] to 35,000 amino acids long. The variance of Titin's count is due to different splice isoforms, which mean different versions of the protein are manufactured by the cell depending on the transcription process at the time of construction. Imagine skipping a word when typing from dictation. In this metaphor the dictation is coming from the DNA coding, and you as the typist are the transcription system, and the resultant written document is the protein amino acid sequence.

3) Fragments of these proteins are called peptides. They can be thought of as syllables or words in the sentence of the protein. When the immune system recognizes the word/syllable in question, that peptide is called an epitope. A chain of amino acids in a certain configuration is recognized by the immune system and is therefore an epitope.

4) The adaptive immune system mounts a specific response to fragments of proteins (epitopes) that are associated with pathogenesis (pathogenicity is the ability for something to cause disease, indicated by failure of innate immune system to vanquish infection).

5) Cross-reactivity occurs when there is enough epitope similarity in either identity (measured in percentage of overlapping amino acids) or physiochemical properties. So either the letters are the same (percent identity), or the meaning of the sentence is same (percent similarity). Both structure (the three dimensional form) and composition (the amino acid sequence) matters for identification. Meaning a 50\% overlap in amino acids that appears very similar to an epitope can be mistaken for the original epitope when a $90 \%$ overlap with a different structure may not. The gold standard of determining similarity is, therefore, experimental.

First, to check for any similarity of the spike glycoprotein (the sequence of characters in our analogy) anywhere in the human body (the set of proteins found in an organism, also known as a proteome), I searched for its sequence (Figure 1) in several databases at the National Center for Biotechnology Information using their Basic Local Alignment Search Tool [7], which runs alignment/similarity queries. There was no significant similarity found in the three databases (RefSeq non-redundant proteins, RefSeq, UniProtKB/Swiss-Prot). Then I specifically pasted the sequences into a tool that would check for alignment between the defined proteins, where both the spike glycoprotein and syncytin-1 (Figure 3) were inputted. Running a search with default parameters again led to no significant similarity, only after manipulating the search parameters to be as loose/sensitive (note that the looser the parameters the higher chance of noise over signal, i.e., spurious hits) as possible (specifically, setting word size to 2, Gap Costs to Existence: 6 Extension: 2, and removing filter for Low complexity regions, adding a Mask for lookup table only) did a similarity emerge (Figure 2).

Second, syncytin- 1 is a protein that is expressed inside the cell. Therefore, an antibody would not interact with it since antibodies circulate outside the cell. An immune response to syncytin- 1 containing cells would therefore have to be mediated by T-cells. The mechanism for this interaction would be:

1) The protein in question (spike or syncitin-1 inside the cell) would be manufactured in the cell.

2) Then the manufactured protein would be cleaved (cut) into fragments (peptides/epitopes) by proteases (protein scissors, in our analogy they would take an entire protein sequence, for example a poem, and cut it into lines of the poem-the lines being peptides/epitopes). 
3) These fragments (peptides/epitopes) enter the endoplasmic reticulum (an organelle within the cell), via transporters, where they are integrated into a major histocompatibility complex (MHC) molecule (think of the MHC as a boat that allows these peptides/epitopes to travel towards the surface of the cell).

4) The MHC-epitope complex (the boat containing the peptide/epitope) is then transferred to the Golgi apparatus.

5) From the Golgi apparatus the MHC-epitope complex is transported to the surface of the cell and is therefore interactive with the extracellular environment (the world surrounding the exterior of the cell).

6) The T-cell would then recognize the epitope in the MHC-epitope complex that was presented on cell surface.

In this mechanism of potential cross-reactivity the epitopes from the spike glycoprotein must have enough similarity to the epitopes in syncytin-1 such that the T-cell would be confused. The predicted epitopes of both the spike glycoprotein and syncitin-1 are listed below (Table 1).

Table 1. Predicted epitopes of syncytin-1 and spike glycoprotein [8]

\begin{tabular}{ll}
\hline Spike glycoprotein & Syncitin-1 \\
\hline MFVFLVLLPLVSSQC & PCIFNLLVNFVSSRI \\
YLYRLFRKSNLKPFE & HIFLFTVLLPSFTLT \\
TRFQTLLALHRSYLT & LLVNFVSSRIEAVKL \\
IWLGFIAGLIAIVMV & SMCFLSFLVPPMTIY \\
LPFFSNVTWFHAIHV & FLGPLAAIILLLLFG \\
AEIRASANLAATKMS & PWILPFLGPLAAIIL \\
TQDLFLPFFSNVTWF & PLNFRPYVSIPVPEQ \\
VFNATRFASVYAWNR & \\
IGINITRFQTLLALH & \\
TDEMIAQYTSALLAG & \\
TNFTISVTTEILPVS & \\
QPYRVVVLSFELLHA & \\
IAGLIAIVMVTIMLC & \\
VVLSFELLHAPATVC & \\
KWPWYIWLGFIAGLI & \\
SIIAYTMSLGAENSV & \\
CPFGEVFNATRFASV & \\
\hline
\end{tabular}

However, a lack of similarity of these epitopes cannot rule out potential for cross-reactivity, since even epitopes with very scant sequence similarity have been known to cross-react. Specifically in the case of myelin basic protein (MBP) epitope cross-reactivity was observed as indicated in the table below (Table 2) [9].

Table 2. Sequence alignment of viral/bacterial mimicry peptides that stimulate MBP-specific $T$ cell clones that are DQ1 or DR2 restricted [9]

\begin{tabular}{ll}
\hline Peptides recognized by clone Hy.1B11 (DO1 restricted) & \\
& $85 \quad 90 \quad 94 \quad 99$ \\
MBP(85-99) & ENPVVHFFK NIVTPR \\
Herpes simplex, UL15 protein & FRQLVHFVRDFAQL \\
Adenovirus type 12, ORF & DFEVVTFLKDVLPEF \\
Pseudomonas, phosphomannomutase & DRLLM LFAKDVVSRN \\
Human papillomavirus type 7, L2 protein & IGGRVHFFKD ISPIA \\
Peptides recognized by clone Hy.1G11 (DR2 restricted) & \\
& $85 \quad 90 \quad 94 \quad 99$ \\
MBP(85-99) & ENPVVHFFKNIVTPR \\
EBV, DNA polymerase & TGGVYHFVKKHVHES \\
Influenza type A, hemagglutinin & YRNLVWFI KKNTRYP \\
Reovirus type 3, sigma 2 protein & MARAAFLF KTVGFGG \\
\hline
\end{tabular}


Table 2. Sequence alignment of viral/bacterial mimicry peptides that stimulate MBP-specific $T$ cell clones that are DQ1 or DR2 restricted [9] (continued)

\begin{tabular}{|c|c|c|}
\hline \multicolumn{3}{|c|}{ Peptides recognized by clone Hy.1G11 (DR2 restricted) } \\
\hline & 94 & 9 \\
\hline $\operatorname{MBP}(85-99)$ & \multicolumn{2}{|l|}{ ENPVV H FFKNIVTPR } \\
\hline EBV, DNA polymerase & \multicolumn{2}{|l|}{ TGGVYHFVKKHVHES } \\
\hline Influenza type A, hemagglutinin & \multicolumn{2}{|l|}{ YRNLVWFI KKNTRYP } \\
\hline Herpes simplex, DNA polymerase & \multicolumn{2}{|l|}{ GGRRLF FVKAHVRES } \\
\hline
\end{tabular}

ORF: open reading frame; EBV: Epstein-Barr virus

Note. Reprinted from "Molecular mimicry in $\mathrm{T}$ cell-mediated autoimmunity: viral peptides activate human $\mathrm{T}$ cell clones specific for myelin basic protein" by Wucherpfennig KW, Strominger JL. Cell. 1995;80:695-705 (https://www.cell.com/cell/pdf/0092-8674(95)90348-8.pdf?_returnURL=https\%3A\%2F\%2Flinkinghub.elsevier. com\%2Fretrieve\%2Fpii\%2F0092867495903488\%3Fshowall\%3Dtrue). @ 1995, Cell Press.

\section{Clinical evidence}

If the claim of cross-reactivity leading to infertility was true (via any mechanism), we should have observed miscarriages at a higher rate among women who contracted the novel coronavirus; as the immune response would be similar to vaccination with additional (non-spike related) causes for concern via other proteins in SARS-CoV-2 (COVID-19) that could have been identified and targeted by the adaptive immune system. Moreover, sterility stemming from vaccination or infection has not been found clinically via an analysis of implantation rates [10].

As per the Centers for Disease Control and Prevention (CDC) [11] data capture of 20 jurisdictions from March 29, 2020 to January 8, 2021, there were 9,545 completed pregnancies from women who tested positive for COVID-19. Of these, 9,466 were live births, and 79 were pregnancy losses (miscarriages). This equates to a $0.83 \%$ rate of miscarriage. As a raw rate, this is not a cause for concern since miscarriage risk drops with pregnancy time [starting from a cumulative incidence estimate of $21.3 \%$ after gestational week 5 [12] and to around 1\% [12] (Figure 4) or less as pregnancy approaches completion]. This is a strong indication that the cross-reactivity leading to infertility via failed placentation claim is false.

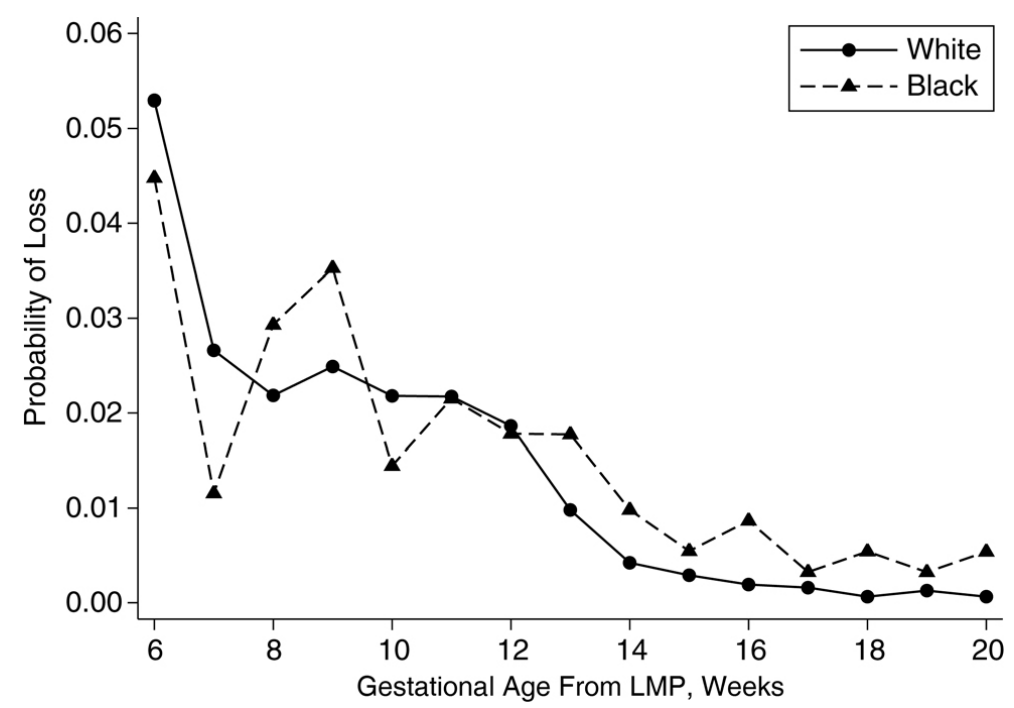

Figure 4. Week-specific probability of pregnancy loss by race among "Right From the Start" participants, 2000-2009. The $x$-axis is gestational age at loss from last menstrual period (LMP); the $y$-axis is probability of pregnancies ending in miscarriage [12] Note. Reprinted from "Risk of miscarriage among black women and white women in a US prospective cohort study" by Mukherjee S, Edwards DRV, Baird DD, Savitz DA, Hartmann KE. Am J Epidemiol. 2013;177:1271-8 (https://academic.oup.com/ aje/article/177/11/1271/97504\#authorNotesSectionTitle). () The Author 2013.

One final defense of the claim would be that, perhaps, the immune response to the protein would only play a significant role in early placentation, while more mature pregnancies would be able to manage the immune attack on the protein, or perhaps even be able to function without it at such a late stage. 
To investigate such a potentiality, I looked again at the most up to date (as of this writing) CDC information on pregnant women with confirmed COVID-19 tests and found that they had collected data on the timing of infection for $98.9 \%(31,158)$ of the women in the cohort, and that of those there were 6217 in the first trimester, with 348 total pregnancy losses (across all trimesters) out of 34,016 outcomes (with 33,668 live births) as of November 5, 2021 [11] (Figure 5). Moreover, there were 23 pregnancies reported during the Pfizer/BioNTech clinical trials with no adverse effects reported, as well as a CDC registry which has enrolled about 15,000 pregnant women as of January 27, 2021 [13] for further investigation and monitoring.

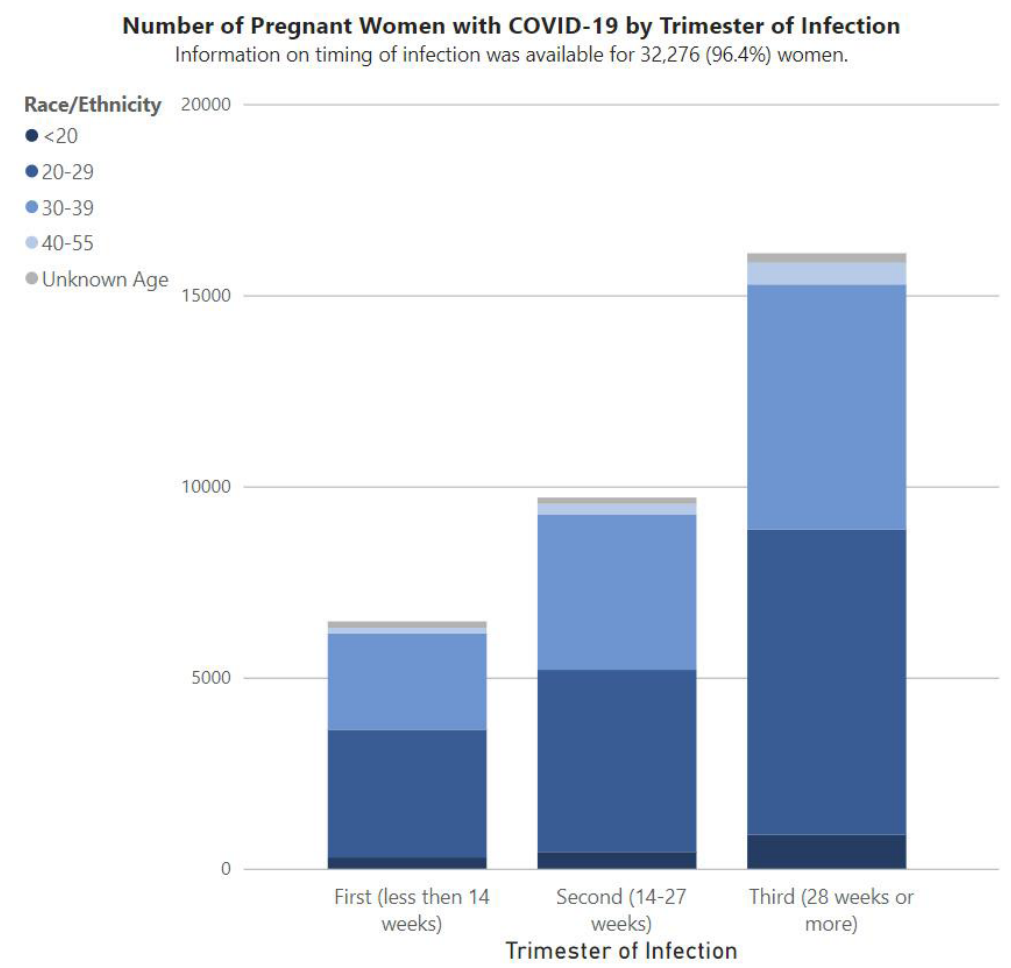

Figure 5. Data reported to CDC as of November 5, 2021 [11]

Note. Reprinted from "Data on COVID-19 during pregnancy: birth and infant outcomes" by CDC. [cited 2021 Feb 11] (https:// covid.cdc.gov/covid-data-tracker/?CDC_AA_refVal=https\%3A\%2F\%2Fwww.cdc.gov\%2Fcoronavirus\%2F2019-ncov\%2Fcasesupdates $\% 2$ Fspecial-populations\%2Fbirth-data-on-covid-19.html\#pregnant-birth-infant). Source: CDC; Materials developed by CDC. Reference to specific commercial products, manufacturers, companies, or trademarks does not constitute its endorsement or recommendation by the U.S. Government, Department of Health and Human Services, or Centers for Disease Control and Prevention. Material is otherwise available on the CDC website for no charge.

\section{Conclusion}

I am very comfortable dismissing the claim since the very basis is not supported by the evidence. To summarize my reasons for rejecting this claim:

1) Antibodies (cross-reactive or otherwise) circulate outside the cell, therefore intracellular protein Syncytin-1 could not cause an immune attack from antibodies.

2) There is no clear similarity between the spike and syncytin-1 proteins, or their epitopes, to indicate cause for concern related to T-cell mediated cross-reactivity.

3) There are many cases of successful pregnancies of women who tested positive for COVID-19 during pregnancy, as well as women who got pregnant during vaccine trials (23 women enrolled in Pfizer/BioNTech COVID-19 vaccine trials got pregnant, 1 suffered miscarriage but was in the control (placebo) group [14]), with no evidence of increased rates of miscarriage.

Absence of evidence is not evidence of absence-there is no way to prove a negative, and only opportunity to demonstrate a lack of basis. For instance, we have systematically identified epitopes in commonly consumed animals and plants that are $100 \%$ identical to epitopes implicated in autoimmune disorders $[15,16]$. These epitopes we can assure are cross-reactive, as their sequences are precisely the 
same. Epitopes whose sequence is not $100 \%$ identical might cross-react or not, so the absence of $100 \%$ identical epitopes cannot rule out the presence of cross-reactive epitopes. One small study on 15 women who were vaccinated showed no anti-syncytin-1 binding antibodies at two time points post vaccination (1-4 days and 4-7 weeks) [17].

There are, and will continue to be, many unsubstantiated claims about COVID-19, vaccines, as well as most health topics that capture the public interest. In the context of decisions related to survival and reproduction such fearmongering claims require strong responses.

I have found no evidence in support of the claim that cross-reactivity of spike glycoprotein antibodies to syncytin-1 cause infertility via failed placentation. I hope that further laboratory experiments will confirm a lack of cross-reactivity, and competent statistical analysis of COVID-positive pregnancies, post-vaccine pregnancies, and control pregnancies will create multiple lines of independent real-world validation.

\section{Abbreviations}

CDC: Centers for Disease Control and Prevention

COVID-19: coronavirus disease 2019

MBP: myelin basic protein

MHC: major histocompatibility complex

SARS-CoV-2: severe acute respiratory syndrome coronavirus-2

\section{Declarations}

\section{Acknowledgements}

I thank Dr. Leonardo MR Ferreira for his invaluable feedback and Sarah Cannon for her editorial assistance. This article is dedicated to the memory of Mark Veksler and Pinhas Bratshpis who died of COVID-19.

\section{Author contributions}

The author contributed solely to the work.

\section{Conflicts of interest}

The author declares that he has no conflicts of interest.

\section{Ethical approval}

Not applicable.

\section{Consent to participate}

Not applicable.

\section{Consent to publication}

Not applicable.

\section{Availability of data and materials}

Not applicable.

\section{Funding}

Not applicable.

\section{Copyright}

(C) The Author(s) 2022. 


\section{References}

1. Former top Pfizer scientist Michael Yeadon claims COVID vaccines may cause infertility in women [Internet]. The European Union Times; c2020 [cited 2021 Aug 24]. Available from: https://www. eutimes.net/2020/12/former-top-pfizer-scientist-michael-yeadon-claims-covid-vaccines-may-causeinfertility-in-women/

2. Comirnaty [Internet]. European Medicines Agency; c1995-2021 [cited 2021 Aug 24]. Available from: https://www.ema.europa.eu/en/medicines/human/EPAR/comirnaty

3. FDA approves first COVID-19 vaccine [Internet]. Food and Drug Administration; c2021 [cited 2021 Aug 24]. Available from: https://www.fda.gov/news-events/press-announcements/fda-approves-firstcovid-19-vaccine

4. Surface glycoprotein [severe acute respiratory syndrome coronavirus 2] [Internet]. National Center for Biotechnology Infirmation; c2021 [cited 2021 Aug 24]. Available from: https://www.ncbi.nlm.nih.gov/ protein/YP_009724390.1

5. Syncytin-1 precursor [Homo sapiens] [Internet]. National Center for Biotechnology Infirmation; c2021 [cited 2021 Aug 24]. Available from: https://www.ncbi.nlm.nih.gov/protein/NP_001124397

6. Titin [Homo sapiens] [Internet]. National Center for Biotechnology Infirmation; c2021 [cited 2021 Aug 24]. Available from: https://www.ncbi.nlm.nih.gov/protein/CAA62188.1

7. Basic Local Alignment Search Tool [Internet]. National Center for Biotechnology Infirmation; c2021 [cited 2021 Aug 24]. Available from: https://blast.ncbi.nlm.nih.gov/Blast.cgi

8. Deimmunization tool [Internet]. IEBD Analysis Resource-Labs; c2021 [cited 2021 Aug 24]. Available from: http://tools.iedb.org/deimmunization/

9. Wucherpfennig KW, Strominger JL. Molecular mimicry in T cell-mediated autoimmunity: viral peptides activate human T cell clones specific for myelin basic protein. Cell. 1995;80:695-705.

10. Morris RS. SARS-CoV-2 spike protein seropositivity from vaccination or infection does not cause sterility. F S Rep. 2021;2:253-5.

11. Data on COVID-19 during pregnancy: birth and infant outcomes [Internet]. Centers for Disease Control and Prevention; [cited 2021 Feb 11]. Available from: https://covid.cdc.gov/covid-data-tracker/?CDC_AA_ refVal=https\%3A\%2F\%2Fwww.cdc.gov\%2Fcoronavirus\%2F2019-ncov\%2Fcases-updates\%2Fspecialpopulations\%2Fbirth-data-on-covid-19.html\#pregnant-birth-infant

12. Mukherjee S, Edwards DRV, Baird DD, Savitz DA, Hartmann KE. Risk of miscarriage among black women and white women in a US prospective cohort study. Am J Epidemiol. 2013;177:1271-8.

13. COVID-19 vaccines and pregnancy: what you need to know if you're pregnant, trying to get pregnant, or breastfeeding [Internet]. UChicagoMedicine; c2021 [cited 2021 Feb 11]. Available from: https://www. uchicagomedicine.org/forefront/coronavirus-disease-covid-19/mrna-covid-19-vaccine-pregnancybreastfeeding

14. Vaccine skeptics' misplaced infertility concerns [Internet]. NATIONAL REVIEW; [cited 2021 Feb 11]. Available from: https://www.nationalreview.com/2021/02/vaccine-skepticsmisplaced-infertility-concerns/

15. Gershteyn IM, Ferreira LMR. Immunodietica: a data-driven approach to investigate interactions between diet and autoimmune disorders. J Transl Autoimmun. 2019;1:100003.

16. Gershteyn IM, Burov AA, Miao BY, Morais VH, Ferreira LMR. Immunodietica: interrogating the role of diet in autoimmune disease. Int Immunol. 2020;32:771-83.

17. Mattar CNZ, Koh W, Seow Y, Hoon S, Venkatesh A, Dashraath P, et al. Addressing anti-syncytin antibody levels, and fertility and breastfeeding concerns, following BNT162B2 COVID-19 mRNA vaccination. medRxiv 21257686 [Preprint]. 2021 [cited 2021 Dec 7]. Available from: https://www.medrxiv.org/ content/10.1101/2021.05.23.21257686v1 